ORIGINAL RESEARCH

Z. Vajda

T. Güthe

M. Aguilar Perez

A. Heuschmid

E. Schmid

H. Bäzner

H. Henkes

\title{
Neurovascular In-Stent Stenoses: Treatment with Conventional and Drug-Eluting Balloons
}

\author{
BACKGROUND AND PURPOSE: ISRs remain a major issue in the endovascular management of ICAD, \\ requiring retreatment by reangioplasty. The aim of the present study was to evaluate the technical \\ feasibility, safety, and efficiency of the novel DEBs for neurovascular ISRs.
}

MATERIALS AND METHODS: Fifty-one patients (median age, 67 years; age range, 34-82 years; male/ female ratio, 37:14) underwent 63 balloon dilation procedures for ISRs in intracranial stented arterial segments between November 2007 and August 2010 in a single center. Of the 63 procedures, 20 $(32 \%)$ were performed by using a conventional balloon and $43(68 \%)$, by using a paclitaxel-eluting balloon (SeQuent Please). Angiographic and clinical follow-up was performed at 6 and 12 weeks, 6 and 12 months, and yearly thereafter. Technical success rate, periprocedural complications, occurrence of recurrent ischemic symptoms, and the development of a recurrent ISR after reangioplasty were analyzed.

RESULTS: Technical success, defined as $<50 \%$ residual stenosis was achieved in all cases (100\%), with failure of the DEB treatment in $6 \%$ of the attempts; those lesions were finally successfully treated with a conventional balloon. The combined permanent neurologic morbidity and mortality rate (stroke, ICH, and $\mathrm{SAH})$ at 30 days was $1.6 \%$. Substantial difference was found in the rate of recurrent stenosis when comparing conventional balloons and DEBs, with recurrent stenosis rates of $50 \%$ and $9 \%$, respectively.

CONCLUSIONS: The initial results of reangioplasty of intracranial ISRs with DEBs are encouraging; further technical developments are, nevertheless, mandatory.

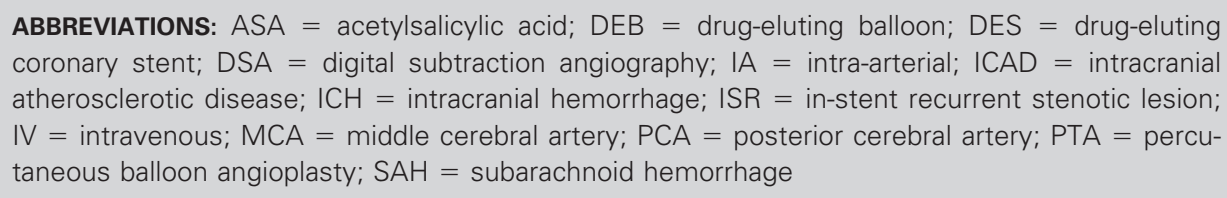

$S_{\text {i }}$ ignificant stenoses $(\geq 50 \%)$ related to ICAD are responsible for approximately $8 \%-10 \%$ of initial ischemic strokes. " "Best medical therapy" with platelet antiaggregation or surgical treatment by extra-to-intracranial bypass gives unsatisfactory results in the management of these patients, ${ }^{2,3}$ driving an emerging interest and rapid technical development in endovascular techniques for the treatment of ICAD.

Early results of PTA without the implantation of a stent showed this method to be an effective treatment for intracranial stenoses ${ }^{4}$; however, issues like dissection and early elastic recoiling still persist, and experience with these procedures is limited. ${ }^{5,6}$

The options for stent treatment in intracranial stenotic disease include the implantation of a balloon-mounted bare metal or drug-eluting stent, originally designed for the coronary vasculature, ${ }^{7,8}$ application of a dedicated neurovascular balloon-mounted stent, ${ }^{9}$ and balloon angioplasty followed by the implantation of a self-expanding stent. ${ }^{10}$

Although the available literature is rather limited at the

Received December 23, 2010; accepted after revision March 5, 2011

From the Klinik für Neuroradiologie (Z.V., T.G., M.A.P., A.H., H.H.), Katharinenhospital and Klinik für Neurologie (E.S., H.B.), Bürgerhospital, Klinikum Stuttgart, Stuttgart, Germany; and Medizinische Fakultät der Universität Duisburg-Essen (H.H.), Essen, Germany.

Please address correspondence to Hans Henkes, MD, Klinik für Neuroradiologie, Katharinenhospital, Klinikum Stuttgart, Kriegsbergstr 60, D-70174 Stuttgart, Germany; e-mail: hhhenkes@aol.com

Indicates open access to non-subscribers at www.ajnr.org

http://dx.doi.org/10.3174/ajnr.A2644 present, the best long-term results have been reported with the application of DESs, with reported hemodynamically significant ISR rates of no more than $5 \%,{ }^{8,11}$ compared with $13.8 \%$ seen with bare metal stents. ${ }^{12}$ The trade-off, however, is the substantially worse initial technical success rate due to the stiffness of the DESs, rendering them frequently unusable in patients with elongated supra-aortic vascular anatomy. Selfexpanding stents (Wingspan, Boston Scientific, Natick, Massachusetts; Enterprise, Cordis, Miami Lakes, Florida), on the other hand, can be delivered in almost every anatomic situation, however, with reported ISR rates of $\leq 31 \%$. $^{13,14}$

ISR with $>50 \%$ luminal loss may again cause cerebral ischemia; therefore, the treatment of these patients is mandatory. ISR typically has been attributed to the proliferation of vascular smooth muscle cells within the stented segment, finally resulting in a hemodynamically significant lumen loss, which is similar to or even worse than the original stenotic lesion. ${ }^{15}$ Traditionally, the treatment option for these lesions was balloon dilation with or without the coaxial implantation of a second stent. This approach yields mostly good immediate results with complete lumen restoration. Angioplasty with a conventional bare surface balloon, however, does not interfere with the underlying pathomechanism of ISR and, therefore, frequently results in a series of several recurrent ISRs and reangioplasties. This occurs frequently in young patients with an initial stenosis of presumably vasculitic origin. ${ }^{16}$ Published data on the treatment of ISR in the neurovascular field are, to our knowledge, sparse.

With the recent introduction of angioplasty balloons 
coated with the antiproliferative drug paclitaxel, it is now possible to restore the narrowed lumen and to biologically interrupt the pathomechanism of ISR at the same time. ${ }^{17}$ Promising initial results have been reported on the application of these DEBs in ISR of the coronary and leg arteries. ${ }^{18,19}$

In the present case series of 51 consecutive patients with high-grade neurovascular ISRs, we compared the technical feasibility, safety, and midterm clinical and angiographic results of 63 balloon angioplasty treatments of intracranial ISR performed with either conventional balloons or DEBs

\section{Materials and Methods}

\section{Patients}

The present series comprises 51 consecutive patients (37 men, 14 women) with a mean age of 67 years (range, 34-82 years) who underwent elective balloon dilation of 63 ISRs during a 34-month period between November 2007 and August 2010 at our institution by or under the supervision of the senior author, according to identical principles of pre-, peri- and postprocedural management.

The nature of their disease; the offered treatment in general; and the proposed off-label intracranial use of a peripheral, conventional coronary, or a coronary drug-eluting balloon with the potential alternatives were explained to all patients. We also explained that high recurrence rates with conventional balloons and device rigidity with DEBs are serious device-related issues and may contribute to procedural failure, complications, and symptomatic vessel occlusion in the midterm course after the procedure. (Within the German jurisdiction, off-label use of medical devices is exempted from prosecution under the following conditions: 1) The patient has a serious (eg, potentially life-threatening) disorder; 2) there is no reasonable or only a less favorable therapeutic alternative; 3 ) available scientific data justify the expectation that, with this device, a better if not successful outcome could be achieved; and 4) the patient or his or her legal representative is informed of the off-label character as well as the procedural risks and potential treatment alternatives.) All patients gave written informed consent to the intervention and follow-up angiographies.

\section{Procedures}

Endovascular treatment was offered to patients with an ISR with the following conditions: 1 ) The stenosis grade was $\geq 50 \%, 2$ ) the lesion was progressive during follow-up (ie, the actual angiographic follow-up revealed a higher grade in-stent stenosis compared with the results of the previous examination), or 3) there were any recurrent neurologic symptoms related to the affected vascular territory. All patients were explicitly advised about the off-label intracranial use of the coronary $\mathrm{DEB}$, including potential anticipated or unknown risks.

The preprocedural management included a neurologic examination, a brain MR imaging/MR angiography, and the administration of a loading dose of $500 \mathrm{mg}$ of ASA and $600 \mathrm{mg}$ of clopidogrel at least 1 day before the treatment. Dual antiplatelet medication was continued after the procedure with $100 \mathrm{mg}$ of ASA and $75 \mathrm{mg}$ of clopidogrel daily for 1 year and $100 \mathrm{mg}$ of ASA daily thereafter.

All endovascular procedures were performed with the patient under general anesthesia via a $6 \mathrm{~F}$ guiding catheter. Standard periprocedural medication included $5000 \mathrm{U}$ of heparin IV, $500 \mathrm{mg}$ of IV ASA, $2 \mathrm{mg}$ of IA glycerin trinitrate, and $1000 \mathrm{mg}$ of IV thiopental, immediately before balloon angioplasty. The target stenosis was visualized

\begin{tabular}{lc}
\hline Table 1: List of the applied balloons & \\
\hline Name (Manufacturer) & No. of Procedures \\
\hline Conventional & 20 \\
Ryujin (Terumo, Tokyo, Japan) & 13 \\
Falcon Bravo (Invatec, Roncadelle, Italy) & 3 \\
Avion Plus (Invatec) & 1 \\
Hiryu (Terumo) & 1 \\
Sprinter Legend (Medtronic, Minneapolis, Minnesota) & 1 \\
SeQuent (B. Braun Melsungen, Berlin, Germany) & 1 \\
Drug-eluting & 43 \\
SeQuent Please (B. Braun Melsungen) & 43 \\
\hline
\end{tabular}

without other overprojecting vessels and with the least possible foreshortening.

Stenosis grades were determined by using the calibrated measurement software of the Axiom Artis DSA system (Siemens, Erlangen, Germany) according to the Warfarin Aspirin Symptomatic Intracranial Disease method. ${ }^{20}$

Via the guiding catheter, the ISR was crossed with a 0.014-inch microguidewire (X-Celerator14, ev3, Irvine, California; or Traxcess14, MicroVention, Aliso Viejo, California) with the tip of the wire placed sufficiently distal to the lesion, allowing a reasonable backup for the balloon crossing the lesion. Over the microguidewire, a properly sized balloon was centered across the lesion and was inflated slowly to nominal pressure for the given diameter with a manometer. The applied balloons are listed in Table 1. DEBs were kept inflated for 1 minute to facilitate drug penetration into the vessel wall. ${ }^{16}$ After deflation and withdrawal of the balloon catheter, a DSA run was performed to confirm the result and to exclude vessel dissection, perforation, or distal embolization. During the first 3-4 days after the procedure, we attempted to maintain the systolic blood pressure at $\leq 130 \mathrm{~mm} \mathrm{Hg}$. Before discharge, all patients underwent MR imaging as well as a clinical examination. Angiographic and clinical follow-up was scheduled for 6 and 12 weeks, 6 and 12 months, and yearly thereafter. The follow-up angiography included DSA runs with projections and magnifications equivalent to those used at the original treatment. On these occasions, patients were clinically examined and specifically interviewed for intermittent transient ischemic events.

\section{Data Collection and Analysis}

Data registry included the following: age and sex of the patients; location of the target lesion; date of the stent implantation; type of the implanted stent; time between the stent implantation and the discovery of the ISR; normal vessel diameter adjacent to the target stenosis; degree of stenosis; type and actual diameter at inflation of the balloon used for dilation of the in-stent stenotic lesion; residual stenosis after dilation; occurrence, type, and severity of procedural complications; minimal in-stent diameter, and occurrence of ischemic signs and symptoms related to the treated stenosis during follow-up. Descriptive statistical methods were applied.

\section{Results}

Fifty-one patients (median age, 67 years; age range, 34-82; male/female ratio, 37:14) underwent 63 balloon dilation procedures for ISRs in 51 intracranial stented arterial segments between November 2007 and August 2010. The lesions were located as follows: internal carotid artery $(n=7,[14 \%])$, MCA $(n=24,[47 \%])$, vertebral artery $(n=15,[29 \%])$, basilar artery $(n=4,[8 \%])$, and PCA $(n=1,[2 \%])$. Thirty-one 


\begin{tabular}{|c|c|c|c|c|c|c|c|}
\hline \multirow[b]{2}{*}{ Balloon Type } & \multirow[b]{2}{*}{$\begin{array}{c}\text { No. of } \\
\text { Procedures }\end{array}$} & \multicolumn{2}{|c|}{$\begin{array}{c}\text { In-Stent } \\
\text { Stenosis Rate }\end{array}$} & \multicolumn{3}{|c|}{ Angiographic Follow-Up } & \multirow[b]{2}{*}{$\begin{array}{c}\text { Lesions } \\
\text { with Recurrent } \\
(\geq 50 \%) \text { Stenosis }\end{array}$} \\
\hline & & $\begin{array}{c}\text { Before } \\
\text { Treatment }\end{array}$ & $\begin{array}{c}\text { After } \\
\text { Treatment }\end{array}$ & $\begin{array}{c}\text { No. of } \\
\text { Lesions with } \\
\text { Follow-Up }(\%)\end{array}$ & $\begin{array}{c}\text { Average } \\
\text { Follow-Up } \\
\text { Period (months) }\end{array}$ & $\begin{array}{c}\text { Maximal } \\
\text { Follow-Up } \\
\text { Period (months) }\end{array}$ & \\
\hline Conventional & 20 & $65 \pm 3 \%$ & $24 \pm 3 \%$ & $18(90 \%)$ & 6.5 & 19 & $50 \%$ \\
\hline Drug-eluting & 43 & $62 \pm 2 \%$ & $24 \pm 2 \%$ & $43(100 \%)$ & 7.5 & 15 & $9 \%$ \\
\hline
\end{tabular}

(60\%) lesions were located in the anterior and $20(40 \%)$, in the posterior circulation.

In-stent balloon dilation was indicated due to the symptomatic occlusion of the stented segment in $1(2 \%)$ patient, a high-grade ( $\geq 50 \%)$ in-stent stenotic lesion verified by angiography in 57 (90\%) patients, recurrent neurologic symptoms attributable to the dependent vascular territory with a non-high-grade recurrent stenotic lesion in $2(3 \%)$ patients, and rapid progression of a non-high-grade ISR in $3(5 \%)$ patients. The severity of the significant $(\geq 50 \%)$ ISR was $50 \%-$ $69 \%$ in $39(68 \%)$ and $70 \%-95 \%$ in $18(32 \%)$ patients. The average degree of target stenoses was determined as $63.3 \pm 2 \%$ before and $23.9 \pm 2 \%$ after the treatment.

A single balloon dilation was performed in $51(81 \%)$ lesions, whereas ISR necessitated a second treatment in 8 lesions $(13 \%)$, a third treatment in 2 lesions (3\%), and a fourth and fifth additional treatment in 1 lesion each (together $3 \%$ ), respectively.

Of the 63 procedures, 20 (32\%) were performed by using a conventional balloon and $43(68 \%)$ by using a DEB (Table 1$)$. Nineteen percent $(n=8)$ of the interventions with a DEB were for the treatment of an ISR that had been treated previously with a conventional balloon.

The median actual balloon diameter at maximal inflation was $2.7 \mathrm{~mm}$.

Technical success, defined as $\leq 50 \%$ residual stenosis at the end of the procedure, was achieved in all patients (100\%). In 4 of 47 cases (8\%), the SeQuent Please (B. Braun Melsungen, Berlin, Germany) DEB could not be navigated through the in-stent stenotic lesion and the treatment of these lesions was finally performed with a conventional balloon.

\section{Complications}

Of the 63 treatments, 59 (93.6\%) were completed without any complication. Major peri- and postprocedural complications were encountered in 2 patients (3\%). One patient had a fatal hyperperfusion ICH 6 hours after the otherwise uneventful procedure. Another patient had an SAH on the third postinterventional day, Hunt and Hess grade I; no treatment was initiated, and the symptoms resolved without sequelae. Access-site adverse events requiring surgery, as minor complications, occurred in $2(3 \%)$ patients. Vasospasm requiring treatment or vessel perforation was not encountered. Thus, the combined permanent neurologic morbidity and mortality rate (stroke, $\mathrm{ICH}$, and $\mathrm{SAH}) 30$ days after the procedure was $1.5 \%(n=1)$.

Angiographic follow-up examinations were performed in 49 (96\%) lesions. The overall average follow-up was 7.2 months, with the longest follow-up being 19 months. The average and maximum follow-up periods were 6.5 and 19

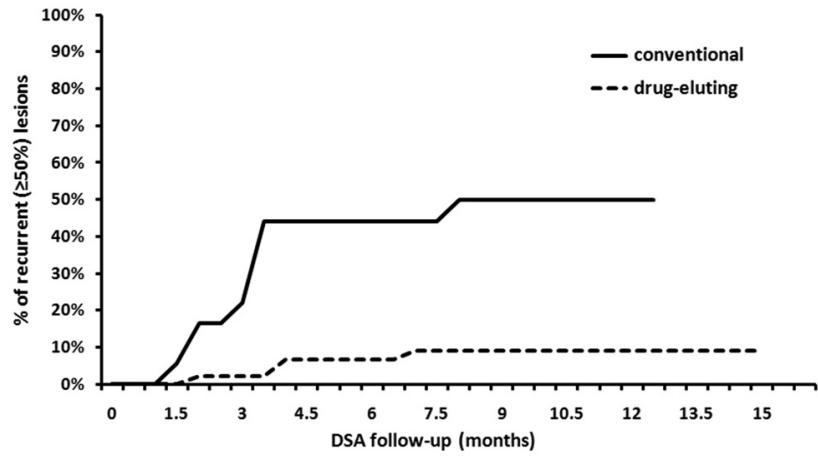

Fig 1. Development of recurrent stenosis following dilation of a high-grade ISR by using conventional balloons (solid line) or DEBs (dashed line), verified by conventional angiography. A high-grade recurrent stenosis (ie, $>50 \%$ luminal loss) developed in $50 \%$ of the lesions treated with conventional balloons after 8 months following the procedure, whereas a substantially lower recurrence rate of $9 \%$ was achieved during the same period by the application of a DEB.

months in lesions dilated with conventional balloons and 7.5 and 14.9 months in lesions treated with DEBs, respectively (Table 2).

Recurrent stenosis following the in-stent balloon dilation, defined as an in-stent stenotic lesion $\geq 50 \%$ on the follow-up DSA series, was reached by 13 of 61 lesions (21\%) after an average follow-up of 3.4 months (Fig 1).

Substantial difference was found in the rate of recurrent stenosis following in-stent balloon dilation with conventional or DEBs, with recurrent stenosis rates of $50 \%$ and $9 \%$, respectively (Table 2 and Fig 1 ).

\section{Discussion}

Stent treatment of significant intracranial stenoses can now be performed with a high level of procedural safety and efficacy. A major drawback is, however, the high incidence of recurrent stenoses resulting from neointimal hyperplasia, which mostly appears within the first 6 months following the stent implantation and frequently shows a rapid progression. The endothelial damage, resulting from the balloon dilation and the electrochemical effects of the implanted metallic stent on the vessel wall, leads to activation of macrophages, smooth muscle cells, and fibroblasts. The activated cells induce the transformation, migration, and proliferation of myofibroblasts, which will gradually cover and thereby insulate the metallic stent struts with neointimal tissue. When this process does not stop at this stage, a rapidly progressive luminal narrowing of the stented segment occurs, finally resulting in a high-grade recurrent stenosis. ${ }^{21}$

It remains a matter of controversy whether and when to retreat patients with ISRs. In most cases, there is a contin- 

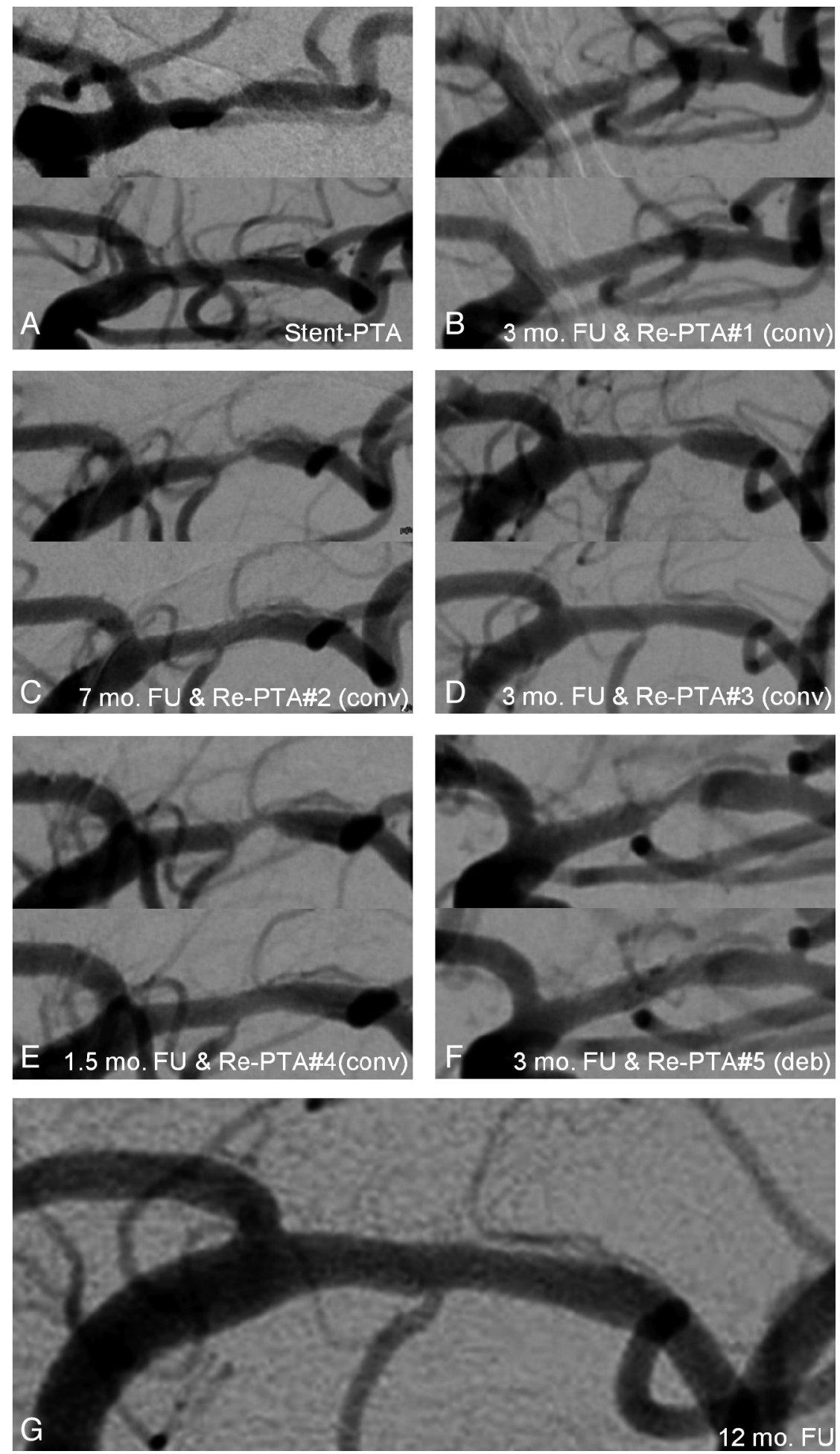

Fig 2. Sequential development of ISRs in 80-year-old male patient with a symptomatic MCA stenosis on the left side. $A$, The stenosis was originally treated with balloon angioplasty and the insertion of an Enterprise stent. $B$, Three months later, DSA follow-up showed high-grade in-stent stenosis, and redilation was performed with a conventional balloon. $C-F$, This was followed by a sequence of recurrent stenotic lesions and reangioplasties with a conventional balloon after $7(C), 3(D), 1.5(E)$, and 3 months $(F)$. $G$, The last reangioplasty was performed by a then-already available DEB, with excellent long-term follow-up results 12 months after the procedure.

uum of progressive luminal loss, which, in our experience, becomes symptomatic at the level of $70 \%-80 \%$ diameter reduction. After a critical threshold of luminal loss has been crossed, the retreatment becomes technically more difficult, and at the same time, the risk of hemodynamic compromise and/or thrombus formation increases. If angio- graphic follow-up shows progression of the intimal hyperplasia, there is little reason to expect spontaneous cessation of this process. For both native and recurrent lesions, the endovascular treatment becomes safer and more controllable if treatment is not postponed to the point when a high-grade stenosis with hemodynamic compromise devel- 
ops. This, in turn, may admittedly result in the treatment of patients who might have had a self-limited or very slowly progressive course of atherosclerosis.

The application of coronary DESs substantially reduces the rate of recurrent stenoses. Their rigidity, however, prohibits the navigation of these stents in tortuous supra-aortic and intracranial vasculature. Moreover, concerns have been raised recently that DESs may be associated with an increased incidence of late thrombotic complications, most likely due to the prolonged endothelization process resulting from the sustained drug release. ${ }^{22}$

The therapeutic option for progressive ISR is angioplasty with or without deployment of a second stent. The treatment of an ISR may be challenging due to difficulties in crossing the fibrous and mostly rigid neointimal plaque within the stented segment, especially when the luminal loss is advanced. The mechanical approach of redilation does not necessarily interfere with the underlying pathophysiologic process and may, therefore, result in second, third, or even more recurrences of neointimal hyperplasia and progressive luminal loss, requiring several retreatments (Fig 2).

The current limitations of the DES technology sparked the development of novel concepts to overcome the sustained drug release from polymers of the stents. In 2004, Scheller et $\mathrm{al}^{17}$ came up with the concept of a balloon catheter as a drug carrier. The antiproliferative drug paclitaxel is fixated with iopromide (Ultravist; Bayer HealthCare Pharmaceuticals, Berlin, Germany) as an enhancer on the surface of a regular angioplasty balloon. Paclitaxel is a potent antiproliferative agent that prevents ISR by inhibiting cell division and migration for weeks and even months, by formation of abnormally stable microtubules. ${ }^{23}$ The physical-chemical properties of the fixation allow only minimal amounts of the drug to be washed away by the blood stream during the insertion of the balloon into the stenotic vessel segment. The drug itself is brought in close contact with the luminal surface of the vessel intima by prolonged balloon inflation for 60 seconds with approximately $16 \%$ of the substance being taken up by the adjacent intima during this period.

\section{In-Stent Restenosis and Re-PTA}

The striking more than 5-fold difference in the occurrence of ISR after angioplasty with paclitaxel-eluting balloons compared with conventional balloons indicates that this technology will have an established place in the inventory of the neurointerventionalist. Nevertheless, technical refinements are warranted. Although technical success, defined as $<50 \%$ luminal narrowing at the end of the procedure, could be achieved in all cases, $8 \%$ of the SeQuent Please DEB attempts failed and those treatments were completed by using a conventional balloon. Being developed on the platform of the coronary SeQuent balloon, the DEB "SeQuent Please" is not optimized for use in intracranial vessels. The distal tip is too short and stiff, and there is not any over-the-wire variant available to date, to our knowledge.

The combined neurologic morbidity and mortality rates of $0 \%$ and $1 \%$, respectively, at 30 days following the intervention in this series of patients is acceptable, especially considering the fact that the patient corresponding to the $1 \%$ mortality was lost due to a fatal reperfusion hemorrhage several hours after the intervention, so the complication was, thereby, not directly related to the mechanical manipulations during the procedure.

\section{Limitations}

This study has several limitations. It presents a consecutive nonselected series of patients without prospective data collection and randomization. Only 1 type of DEB currently available on the market was used in this series; therefore, differences in balloon design and drug types cannot be evaluated. The lack of a dedicated neurovascular DEB creates a bias in the comparison of technical success rates between conventional balloons and DEBs. The heterogeneity of pathophysiology (atherosclerotic, vasculitic, and so forth, stenoses) and localization (extra- versus intracranial) of the treated stenoses also limit the evaluation of the results.

\section{Conclusions}

This study provides promising, albeit preliminary, data on the effectiveness of DEBs in the treatment of neurovascular ISR. More flexible balloons with hydrophilic shaft coating are certainly needed to improve the technical success rate.

\section{References}

1. Levy EI, Turk AS, Albuquerque FC, et al. Wingspan in-stent restenosis and thrombosis: incidence, clinical presentation, and management. Neurosurgery 2007;61:644-50

2. Chimowitz MI, Lynn MJ, Howlett-Smith H, et al. Comparison of warfarin and aspirin for symptomatic intracranial arterial stenosis. N Engl J Med 2005;352: 1305-16

3. Failure of extracranial-intracranial arterial bypass to reduce the risk of ischemic stroke: results of an international randomized trial-the EC/IC Bypass Study Group. N Engl J Med 1985;313:1191-200

4. Sundt TM Jr, Smith HC, Campbell JK, et al. Transluminal angioplasty for basilar artery stenosis. Mayo Clin Proc 1980;55:673-80

5. Marks MP, Wojak JC, Al-Ali F, et al. Angioplasty for symptomatic intracranial stenosis: clinical outcome. Stroke 2006;37:1016-20

6. Berg-Dammer E, Henkes H, Weber W, et al. Percutaneous transluminal angioplasty of intracranial artery stenosis: clinical results in 24 patients. Neurosurg Focus 1998;5:e13

7. Vajda Z, Miloslavski E, Güthe T, et al. Treatment of intracranial atherosclerotic arterial stenoses with a balloon-expandable cobalt chromium stent (Coroflex Blue): procedural safety, efficacy, and midterm patency. Neuroradiology 2010;52:645-51. Epub 2009 Nov 17

8. Gupta R, Al-Ali F, Thomas AJ, et al. Safety, feasibility, and short-term follow-up of drug-eluting stent placement in the intracranial and extracranial circulation. Stroke 2006;37:2562-66

9. Mocco J, Darkhabani Z, Levy EI. Pharos neurovascular intracranial stent: elective use for a symptomatic stenosis refractory to medical therapy. Catheter Cardiovasc Interv 2009;74:642-46

10. Henkes H, Miloslavski E, Lowens S, et al. Treatment of intracranial atherosclerotic stenoses with balloon dilatation and self-expanding stent deployment (Wingspan). Neuroradiology 2005;47:222-28. Epub 2005 Mar 15

11. Abou-Chebl A, Bashir Q, Yadav JS. Drug-eluting stents for the treatment of intracranial atherosclerosis: initial experience and midterm angiographic follow-up. Stroke 2005;36:e165-68

12. Gröschel K, Schnaudigel S, Pilgram SM, et al. A systematic review on outcome after stenting for intracranial atherosclerosis. Stroke 2009;40:e340-47

13. Turk AS, Levy EI, Albuquerque FC, et al. Influence of patient age and stenosis location on Wingspan in-stent restenosis. AJNR Am J Neuroradiol 2008;29:23-27

14. Vajda Z, Schmid E, Güthe T, et al. The modified Bose-method for the endovascular treatment of intracranial atherosclerotic arterial stenoses using the Enterprise stent. Neurosurgery 2011 July 19.

15. Scott NA. Restenosis following implantation of bare metal coronary stents: pathophysiology and pathways involved in the vascular response to injury. Adv Drug Deliv Rev 2006;58:358-76

16. Güthe T, Miloslavski E, Vajda Z, et al. Recurrent in-stent restenosis in a symptomatic 
non-atherosclerotic M1 plaque: successful treatment with paclitaxel-eluting balloon dilatation after repeated failure of conventional balloon reangioplasty. Clin Neuroradiol 2010;20:165-69. Epub 2010 May 29

17. Scheller B, Speck U, Abramjuk C, et al. Paclitaxel balloon coating, a novel method for prevention and therapy of restenosis. Circulation 2004;110:81014. Epub 2004 Aug 9

18. Scheller B, Hehrlein C, Bocksch W, et al. Two year follow-up after treatment of coronary in-stent restenosis with a paclitaxel-coated balloon catheter. Clin Res Cardiol 2008;97:773-81

19. Tepe G, Zeller T, Albrecht T, et al. Local delivery of paclitaxel to inhibit reste nosis during angioplasty of the leg. N Engl J Med 2008;358:689-99

20. Samuels OB, Joseph GJ, Lynn MJ, et al. A standardized method for measuring intracranial arterial stenosis. AJNR Am J Neuroradiol 2000;21:643-46

21. Apisarnthanarax S, Chougule P. Intravascular brachytherapy: a review of the current vascular biology. Am J Clin Oncol 2003;26:e13-21

22. Daemen J, Serruys PW. Drug-eluting stent update 2007. Part II. Unsettled issues. Circulation 2007;116:961-68

23. Schachner T, Steger C, Heiss S, et al. Paclitaxel treatment reduces neointimal hyperplasia in cultured human saphenous veins. Eur J Cardiothorac Surg 2007; 32:906-11 